\title{
Common Belief Foundations of Global Games*
}

\author{
Stephen Morris \\ Princeton University
}

\author{
Hyun Song Shin \\ Bank for International Settlements
}

Muhamet Yildiz

M.I.T.

November 2015

\begin{abstract}
We study coordination games under general type spaces. We characterize rationalizable actions in terms of the properties of the belief hierarchies and show that there is a unique rationalizable action played whenever there is approximate common certainty of rank beliefs, defined as the probability the players assign to their payoff parameters being higher than their opponents'. We argue that this is the driving force behind selection results for the specific type spaces in the global games literature.
\end{abstract}

\section{Introduction}

Complete information games often have many equilibria. Even when they have a single equilibrium, they often have many actions that are rationalizable, and are therefore consistent with common certainty of rationality. The inability of theory to make a prediction is problematic for economic applications of game theory.

Carlsson and van Damme (1993) suggested a natural perturbation of complete information that gives rise to a unique rationalizable action for each player. They introduced the idea of "global games" —where any payoffs of the game are possible and each player observes the true payoffs of the game with a small amount of noise. They showed - for the case of two player two action games - that as the noise about payoffs becomes small, there is a unique equilibrium; moreover, the perturbation selects a particular equilibrium (the risk-dominant one) of the underlying game. This result has since been generalized in a number of

${ }^{*}$ This paper incorporates material from a working paper of the same title circulated in 2007 (Morris and Shin (2007)). The first two authors are grateful to the National Science Foundation Grant SES-0648806 for funding this research. We are grateful for comments from editors, anonymous referees, and seminar participants at Columbia, Iowa State, Northwestern, and UCLA on this iteration of the project. We thank Anton Tsoy for detailed comments. The views expressed in this paper reflect those of the authors and not necessarily those of the Bank for International Settlements.

(C) 2016. This manuscript version is made available under the Elsevier user license http://www.elsevier.com/open-access/userlicense/1.0/ 
directions and widely used in applications. ${ }^{1}$ When the global game approach can be applied to more general games, it can be used to derive unique predictions in settings where the underlying complete information game has multiple equilibria, making it possible to carry out comparative static and policy analysis. It has been informally argued that multiplicity partly relies on the unrealistic "complete information" assumption, and the natural perturbation underlying global games captures the more realistic case.

However, the global game selection result uses a particular form of perturbation away from "complete information." Complete information entails the assumption that a player is certain of the payoffs of the game, certain that other players are certain, and so on. Weinstein and Yildiz (2007) consider more general perturbations, saying that a situation is close to a complete information game if players are almost certain that payoffs are close to those complete information game payoffs, almost certain that other players are almost certain that payoffs are close to those payoffs, and so on. Formally, they consider closeness in the product topology on the universal belief space. They show that for any action which is rationalizable for a player in a complete information game, there exists a nearby type of that player in the product topology for whom this is the unique rationalizable action. Thus by considering a richer but also intuitive class of perturbations, they replicate the global game uniqueness result but reverse the selection result.

In this paper, we identify the driving force behind global game uniqueness and selection results. In particular, we do not want to take literally the (implicit) assumption in global games that there is common certainty among the players of a technology which generates (conditionally independent) noisy signals observed by the players. Rather, we want to argue that global game perturbations are a metaphor, or a convenient modelling device, for a more general intuitive class of relaxations of common certainty. We want to characterize and analyze the key property of that more general class, which must also be more restrictive than the product topology perturbations of Weinstein and Yildiz (2007).

Our baseline analysis is carried out for a two player, two action game. Each player must decide whether to "invest" or "not invest". Payoffs are given by the following matrix:

\begin{tabular}{|c|c|c|}
\hline & invest & not invest \\
\hline invest & $x_{1}, x_{2}$ & $x_{1}-1,0$ \\
\hline not invest & $0, x_{2}-1$ & 0,0 \\
\hline
\end{tabular}

Each player $i$ knows his own payoff parameter, or return to investment, $x_{i}$ but may not know the other player's payoff parameter. There are strategic complementarities, because a player has a loss of 1 if the other player does not invest. If $x_{1}$ and $x_{2}$ are both in the interval [0,1], then there are multiple equilibria,

\footnotetext{
${ }^{1}$ Morris and Shin (1998) analyzed a global game with a continuum of players making binary choices, and this case has been studied in a number of later applications. See Morris and Shin (2003b) for an early survey of some theory and applications of global games. Frankel, Morris, and Pauzner (2003) study global game selection in general games with strategic complementarities.
} 
both invest and both not invest, under complete information. In the symmetric case, with $x_{1}=x_{2}=x$, the risk-dominant equilibrium in this game is the equilibrium that has each player choose a best response to a 50/50 distribution over his opponent's action. Thus both invest is the risk-dominant equilibrium if $x>\frac{1}{2}$.

If $x_{1}$ and $x_{2}$ are both in the interval [0,1], both actions remain rationalizable for player $i$ if there is approximate common certainty of payoffs. ${ }^{2}$ This is a well known sufficient condition for multiple rationalizable actions, going back to Monderer and Samet (1989). But it is a strong condition.

A key concept to understand uniqueness in this setting is a player's "rank belief" — that is, his belief about whether he has the higher payoff parameter (so he has rank 1) or the lower payoff parameter (so he has rank 2). A player has the uniform rank belief if he assigns probability $\frac{1}{2}$ to each rank. If there is common certainty of uniform rank beliefs, then a player has a unique rationalizable action. In particular, action invest is uniquely rationalizable for a player if it is risk-dominant.

A rough argument for this is as follows. Let $x^{*}$ be the smallest payoff parameter such that invest is uniquely rationalizable whenever there is common certainty of uniform rank beliefs for a player with payoff parameter greater than or equal to $x^{*}$. If $x^{*}$ were strictly greater than $\frac{1}{2}$, a player with payoff parameter close to $x^{*}$ (and common certainty of uniform rank beliefs) would assign probability close to $\frac{1}{2}$ to his opponent investing and would therefore have a strict incentive to invest. Thus we would have a contradiction. Thus $x^{*}$ must be less than or equal to $\frac{1}{2}$. This proves the result in the standard case with one dimensional types.

Under some additional continuity assumptions, essentially this argument goes though for general situations - without reference to noisy signals or one dimensional type spaces - using the properties of the belief hierarchy at hand. This is how our result provides a primitive common belief foundation for global game selection. One of our main results will be a formalization of an appropriate weakening of the above sufficient condition: approximate common certainty of approximately uniform rank beliefs. And, more generally, the common belief foundations results focus attention on the properties of higher-order beliefs that matter for global game results rather than the conditionally independent noisy signal story that generates them.

Our characterization of rationalizability in terms of higher-order beliefs provides a conceptual foundation for global games approach, making explicit certain intuitions present in the existing literature, as well as extending the approach to richer and possibly more interesting type spaces. The applications in global games literature have been mainly confined to unidimensional type spaces with either an approximately uniform prior or a monotone supermodular structure (e.g. players' signals have a common shock and an idiosyncratic shock - both distributed normally). In such type spaces it suffices to focus on equilibria with

\footnotetext{
${ }^{2}$ There is approximate common certainty of an event if both believe it with probability close to 1 , both believe with probability close to 1 that both believe it with probability close to 1 , and so on.
} 
cutoff strategies. One can study those equilibria directly without needing our machinery here - although the rank beliefs clearly play an important role in those equilibria. Unfortunately, such a direct approach does not extend to higher-dimensional types spaces, where monotone equilibria would have higher-dimensional boundaries, and to the type spaces without monotone supermodular structure, so that monotone equilibria may not even exist. One can still apply our sufficient conditions to those models and find out whether the risk-dominance gives the unique rationalizable solution. We illustrate this point in two examples modifying the standard normality assumptions. In one of them, we simply alter the distribution of variables to Pareto distributions (which often arise when there is a model uncertainty). In this example, monotonicity properties fail but our results still lead to a sharp dichotomy: the risk-dominant action is uniquely rationalizable when the idiosyncratic shock has a thinner tail, and both actions are rationalizable when the common shock has a thinner tail. This sharply contrasts with the normal example, where one needs a peculiar rate of convergence, on which many existing applications are built. In the second example, we simply introduce uncertainty about variances, so that a player does not know how much the other player knows, resulting in a two-dimensional type space. We cannot solve for equilibria in this game, but we can easily extend the conclusions from the standard exercise to this case, by applying our results.

We present our results in the context of the simple strategic setting described above and focussing on uniform rank beliefs. We do this in order to focus on the common belief foundations rather than the details of the strategic setting. We can then describe how our results can be mapped back to more general settings.

Our results extend to the case where there is approximate common certainty of the approximate rank belief $p$, where $p \neq \frac{1}{2}$. The selected action will then depend on the rank belief $p$. In doing so, we provide common belief foundations for the results of Izmalkov and Yildiz (2010). While this case of $p \neq \frac{1}{2}$ has typically been motivated by non-common priors, we will show that it is possible to have approximate common certainty of the rank belief $p$ for any $p \neq \frac{1}{2}$, even under the common prior assumption.

We state our results for symmetric two player two action games, but they extend to symmetric many player two action games. The common belief characterization of rationalizability becomes more complex, because the relevant belief operators depend on beliefs about more than one event. However, the uniqueness and multiplicity results extend cleanly. Common certainty of rank beliefs corresponds in the $N$ player case to always assigning probability $\frac{1}{N}$ to exactly $n$ players having higher payoff states, for each $n=0,1, \ldots, N-1$. Morris and Shin (2003b) dubbed this the Laplacian assumption, and in this sense we are formalizing a known intuition about global game selection. This selection is key in the vast majority of applied work using global games.

However, the global game selection results reported in the paper rely on binary actions and symmetric payoffs; in particular, it is required for common certainty of approximately uniform rank beliefs to be the relevant sufficient condition for unique rationalizable actions. Higher-order belief foundations can be 
provided for many action and asymmetric payoff global game results, but they are qualitatively different from those provided for symmetric two action games here.

We present the basic definitions and preliminary results in Section 2. In Section 3, we present our characterization of rationalizability in terms of higher-order beliefs. In Section 4, we report sufficient conditions for multiple rationalizable outcomes based on approximate common certainty of payoffs and for unique rationalizable outcomes based on approximate common certainty of approximately uniform rank beliefs. In Section 5, we report the proof of the main result as well as an example showing that a key technical assumption is not superfluous. In Section 6, we discuss extensions to more general strategic settings and the relation to the global games literature.

\section{Model}

There are two players, 1 and 2. Let $T_{1}$ and $T_{2}$ be the sets of types for players 1 and 2 , respectively. A mapping $x_{i}: T_{i} \rightarrow \mathbb{R}$ describes a payoff parameter of interest to player $i$, and a mapping $\pi_{i}: T_{i} \rightarrow \Delta\left(T_{j}\right)$ describes player $i$ 's beliefs about the other player. We assume that $T_{i}$ is endowed with a metrizable topology and Borel sigma-algebra, under which the mappings $x_{i}$ and $\pi_{i}$ are measurable. We make a couple of minimal continuity assumptions: $x_{i}$ and $\pi_{i}$ are continuous, ${ }^{3}$ and the pre-image $x_{i}^{-1}([a, b])$ of every compact interval $[a, b]$ is sequentially compact. This type space can be arbitrarily rich, and in particular can encode any beliefs and higher-order beliefs, and thus our results apply if the type space is the universal (private value) belief space of Mertens and Zamir (1985). ${ }^{4}$

We start by describing the belief and common belief operators, as in Monderer and Samet (1989). The state space is $T=T_{1} \times T_{2}$. An event is a subset of $T$. An event is simple if $E=E_{1} \times E_{2}$ where $E_{i} \subseteq T_{i}$. For our game theoretic analysis, we will be interested in simple events and we restrict attention to such events in the analysis that follows. For any such simple event $E$, we write $E_{1}$ and $E_{2}$ for the projections of $E$ onto $T_{1}$ and $T_{2}$, respectively. Now, for probability $p_{i}$, write $B_{i}^{p_{i}}(E)$ for the set of states where player $i$ believes $E$ with probability at least $p_{i}$ :

$$
B_{i}^{p_{i}}(E)=\left\{\left(t_{1}, t_{2}\right) \mid t_{i} \in E_{i} \text { and } \pi_{i}\left(E_{j} \mid t_{i}\right) \geq p_{i}\right\} .
$$

\footnotetext{
${ }^{3}$ We use the standard definition for the continuity of the beliefs: as $t_{i, m} \rightarrow t_{i}, \pi_{i}\left(\cdot \mid t_{i, m}\right)$ converges to $\pi_{i}\left(\cdot \mid t_{i}\right)$ under the weak topology on the probability distributions (as in the "convergence in distribution"). That is, the expectation of any continuous and bounded function under $\pi_{i}\left(\cdot \mid t_{i, m}\right)$ converges to its expectation under $\pi_{i}\left(\cdot \mid t_{i}\right)$. Our results are also valid under an alternative notion of continuity: $\pi_{i}\left(T_{j}^{\prime} \mid \cdot\right)$ is continuous for every measurable subset $T_{j}^{\prime} \subseteq T_{j}$.

${ }^{4}$ Mertens and Zamir (1985) constructed a space that encodes all beliefs and higher-order beliefs about a common state space. We maintain the assumption that the state space is a pair of payoff types of the players, where each player knows his own payoff type. It is a simple adaption to the classic construction to build in this restriction, see for example Heifetz and Neeman (2006). The classical construction assumes a compact state space. We need to allow the state space to be $\mathbb{R}^{2}$ but instead impose sequential compactness of types with payoffs within a compact interval.
} 
For a pair of probabilities $\left(p_{1}, p_{2}\right)$, say that event $E$ is $\left(p_{1}, p_{2}\right)$-believed if each player $i$ believes event $E$ with probability at least $p_{i}$. Writing $B_{*}^{p_{1}, p_{2}}(E)$ for the set of states where $E$ is $\left(p_{1}, p_{2}\right)$-believed, we have:

$$
B_{*}^{p_{1}, p_{2}}(E)=B_{1}^{p_{1}}(E) \cap B_{2}^{p_{2}}(E) .
$$

Say that there is common $\left(p_{1}, p_{2}\right)$-belief of event $E$ if it is $\left(p_{1}, p_{2}\right)$-believed, it is $\left(p_{1}, p_{2}\right)$-believed that it is $\left(p_{1}, p_{2}\right)$-believed, and so on. We write $C^{p_{1}, p_{2}}(E)$ for set of states at which $E$ is common $\left(p_{1}, p_{2}\right)$-belief. Thus

$$
C^{p_{1}, p_{2}}(E)=\bigcap_{n \geq 1}\left[B_{*}^{p_{1}, p_{2}}\right]^{n}(E) .
$$

An event is $\left(p_{1}, p_{2}\right)$-evident if it is $\left(p_{1}, p_{2}\right)$-believed whenever it is true. Generalizing a characterization of common knowledge by Aumann (1976), Monderer and Samet (1989) characterizes common $\left(p_{1}, p_{2}\right)$ beliefs through $\left(p_{1}, p_{2}\right)$-evident events. This characterization is stated along with other useful facts next (see the proof of Lemma 2 below for a proof).

Lemma 1 (Monderer and Samet (1989)) The following are true for all simple events E:

1. $C^{\left(p_{1}, p_{2}\right)}(E) \subseteq \cdots \subseteq\left[B_{*}^{\left(p_{1}, p_{2}\right)}\right]^{n+1}(E) \subseteq\left[B_{*}^{\left(p_{1}, p_{2}\right)}\right]^{n}(E) \subseteq \cdots \subseteq B_{*}^{\left(p_{1}, p_{2}\right)}(E) \subseteq E$.

2. $C^{\left(p_{1}, p_{2}\right)}(E)$ is the largest $\left(p_{1}, p_{2}\right)$-evident event $F$ with $F \subseteq E$.

3. If $E$ is closed, so are $C^{\left(p_{1}, p_{2}\right)}(E)$ and $B_{*}^{\left(p_{1}, p_{2}\right)}(E)$.

In our formulation, we make the belief operators type dependent, and the above properties generalize immediately to this case. For any $f_{i}: T_{i} \rightarrow \mathbb{R}$, we say that event $E$ is $f_{i}$-believed by type $t_{i}$ of player $i$ if he believes it with probability at least $f_{i}\left(t_{i}\right)$ :

$$
B_{i}^{f_{i}}(E)=\left\{\left(t_{1}, t_{2}\right) \mid t_{i} \in E_{i} \text { and } \pi_{i}\left(E_{j} \mid t_{i}\right) \geq f_{i}\left(t_{i}\right)\right\} .
$$

Clearly we can make richer statements about beliefs and higher-order beliefs in this language. We will continue to write $B_{i}^{p_{i}}(E)$ for the original $p_{i}$-belief operator, where $p_{i}$ is now understood as the constant function of types taking the value $p_{i}$. Note that we allow $f_{i}$ to take values below 0 and above 1 . This convention gives a special role to the events $B_{i}^{f_{i}}(\varnothing)$ and $B_{i}^{f_{i}}(T)$, since a player always believes an event with probability at least 0 and never believes an event with probability greater than 1 . Thus

$$
\begin{aligned}
B_{i}^{f_{i}}(\varnothing) & =\left\{\left(t_{1}, t_{2}\right) \mid f_{i}\left(t_{i}\right) \leq 0\right\} \\
B_{i}^{f_{i}}(T) & =\left\{\left(t_{1}, t_{2}\right) \mid f_{i}\left(t_{i}\right) \leq 1\right\} .
\end{aligned}
$$

These operators behave just like the type-independent ones. In particular, writing $B_{*}^{f_{1}, f_{2}}(E)$ for the set of states where $E$ is $\left(f_{1}, f_{2}\right)$-believed, we have:

$$
B_{*}^{f_{1}, f_{2}}(E)=B_{1}^{f_{1}}(E) \cap B_{2}^{f_{2}}(E) .
$$


Say that there is common $\left(f_{1}, f_{2}\right)$-belief of event $E$ if it is $\left(f_{1}, f_{2}\right)$-believed, it is $\left(f_{1}, f_{2}\right)$-believed that it is $\left(f_{1}, f_{2}\right)$-believed, and so on. We write $C^{f_{1}, f_{2}}(E)$ for set of states at which $E$ is common $\left(f_{1}, f_{2}\right)$-belief:

$$
C^{f_{1}, f_{2}}(E)=\underset{n \geq 1}{\cap}\left[B_{*}^{f_{1}, f_{2}}\right]^{n}(E)
$$

An event is $\left(f_{1}, f_{2}\right)$-evident if it is $\left(f_{1}, f_{2}\right)$-believed whenever it is true. Lemma 1 generalizes to our case as follows.

Lemma 2 The following are true for all simple events E.

1. $C^{\left(f_{1}, f_{2}\right)}(E) \subseteq \cdots \subseteq\left[B_{*}^{\left(f_{1}, f_{2}\right)}\right]^{n+1}(E) \subseteq\left[B_{*}^{\left(f_{1}, f_{2}\right)}\right]^{n}(E) \subseteq \cdots \subseteq B_{*}^{\left(f_{1}, f_{2}\right)}(E) \subseteq E$.

2. $C^{\left(f_{1}, f_{2}\right)}(E)$ is the largest $\left(f_{1}, f_{2}\right)$-evident event $F$ with $F \subseteq E$.

3. Assume $f_{1}$ and $f_{2}$ are continuous. If $E$ is closed, so are $C^{\left(f_{1}, f_{2}\right)}(E)$ and $B_{*}^{\left(f_{1}, f_{2}\right)}(E)$.

A proof of this Lemma in this context and notation - following standard arguments - is reported in the Appendix.

In the baseline model, we consider the following action space and payoff function:

\begin{tabular}{|l|c|c|}
\hline & invest & not invest \\
\hline invest & $x_{1}, x_{2}$ & $x_{1}-1,0$ \\
\hline not invest & $0, x_{2}-1$ & 0,0 \\
\hline
\end{tabular}

Note that any two-player two-action game with a pure-strategy Nash equilibrium is best-response equivalent to such a game, so that payoffs can be normalized into payoffs of this form without changing strategic behavior. Player $i$ knows his own payoff parameter $x_{i}\left(t_{i}\right)$ but does not necessarily know the other player's payoff parameter $x_{j}\left(t_{j}\right)$. Moreover, he gets a return $x_{i}\left(t_{i}\right)$ if he invests but faces a penalty 1 if the other player does not invest. Hence, he only wants to invest if the probability he assigns to his opponent investing is at least $1-x_{i}\left(t_{i}\right)$.

Remark 1 In our general setup and in the above payoff function, we have a "private value" environment, in which a player knows his payoff function at the interim stage. ${ }^{5}$ In contrast, a "common-value" environment is often used in global games literature by specifying the payoff function as

\begin{tabular}{|l|c|c|}
\hline & invest & not invest \\
\hline invest & $\theta, \theta$ & $\theta-1,0$ \\
\hline not invest & $0, \theta-1$ & 0,0 \\
\hline
\end{tabular}

\footnotetext{
${ }^{5}$ See Morris and Shin (2005) for some theory and Argenziano (2008) for an application of private value global games.
} 
where the payoff parameter $\theta$ is unknown. This environment is also included in our model by taking

$$
x_{i}\left(t_{i}\right)=E\left[\theta \mid t_{i}\right] .
$$

In both the common-value formulation and the private-value formulation with $x_{i}\left(t_{i}\right)=E\left[\theta \mid t_{i}\right]$ a player invests if and only if the probability he assigns to his opponent investing is at least $1-x_{i}\left(t_{i}\right)$. This bestresponse equivalence leads to strategic equivalence under many solution concepts, including rationalizability and Bayesian Nash equilibrium. We prefer the private value formulation because it leads to a more direct and clearer analysis. Also, in applications in which one studies the limit properties of the solution (as in the examples at the end of Section 4), the private value formulation avoids difficulties related to the convergence of expectations $E\left[\theta \mid t_{i}\right]^{6}$

Throughout the paper, we will use rationalizability as the solution concept. We define rationalizability in the context of this game as follows (it corresponds to standard general definitions). Say that an action is $(k+1)$ th-level rationalizable if it is a best response to $k$ th-level rationalizable play of his opponent; and say that any action is 0th-level rationalizable. Write $R_{i}^{k}$ for the set of types of player $i$ for whom action invest is $k$ th-level rationalizable and let $R_{i}^{0}=T_{i}$.

\section{Common-Belief Characterization of Rationalizability}

In this Section, we provide a useful characterization of rationalizability in terms of higher-order beliefs about the payoffs. We start with carefully describing the set $R_{i}^{1}$ of types for whom invest is 1 st-level rationalizable in terms of our type-dependent belief operators. On the one hand, for any type $t_{i}$ of player $i$, action invest is 1st-level rationalizable for $t_{i}$ if and only if $x_{i}\left(t_{i}\right) \geq 0$; this is in response to the belief that his opponent invests with probability 1 . On the other hand, since player $i$ always assigns probability 1 to $T$, he assigns probability at least $1-x_{i}\left(t_{i}\right)$ to $T$ if and only if $x_{i}\left(t_{i}\right) \geq 0$. Thus,

$$
R_{i}^{1}=B_{i}^{1-x_{i}}(T)
$$

That is, the types for which invest is first-level rationalizable coincide with those in $B_{i}^{1-x_{i}}(T)$.

Now, action invest is 2nd-level rationalizable for type $t_{i}$ of player $i$ if, in addition, he assigns probability at least $1-x_{i}\left(t_{i}\right)$ to $x_{j}\left(t_{j}\right) \geq 0$; thus

$$
R_{i}^{2}=B_{i}^{1-x_{i}}\left(B_{*}^{1-x_{1}, 1-x_{2}}(T)\right) .
$$

\footnotetext{
${ }^{6}$ An additional simplifying assumption here is that payoffs are additively separable between a component that depends on the opponent's action and a component that depends on an unknown payoff state. This additive separability allows for a tighter description of the connection. Extensions are possible here also, but are messy and involve tedious continuity arguments. Also, like much of the applied literature, we focus on a game which is symmetric across players. However, global game results go through with asymmetric games. One can state analogous higher-order belief properties driving global game results (relating to translation invariance) but they are not as clean. These issues are discussed in Morris and Shin (2007).
} 
More generally, action invest is $(k+1)$ th-level rationalizable for a type $t_{i}$ if he $\left(1-x_{i}\left(t_{i}\right)\right)$-believes that $T$ is $k$ th-order $\left(1-x_{1}, 1-x_{2}\right)$-believed:

$$
R_{i}^{k+1}=B_{i}^{1-x_{i}}\left(\left[B_{*}^{1-x_{1}, 1-x_{2}}\right]^{k}(T)\right) .
$$

Action invest is rationalizable if it is $k$ th level rationalizable for all $k$. Thus, action invest is rationalizable for both players exactly if $T$ is common $\left(1-x_{1}, 1-x_{2}\right)$-believed:

$$
R^{\infty}=C^{1-x_{1}, 1-x_{2}}(T)
$$

By a symmetric argument, action not invest is rationalizable exactly if $T$ is common $\left(x_{1}, x_{2}\right)$-belief. The next result states this characterization; recall that $C^{1-x_{1}, 1-x_{2}}(T)=C_{1}^{1-x_{1}, 1-x_{2}}(T) \times C_{2}^{1-x_{1}, 1-x_{2}}(T)$.

Proposition 1 Action invest is rationalizable for type $t_{i}$ if and only if

$$
t_{i} \in C_{i}^{1-x_{1}, 1-x_{2}}(T)
$$

action not invest is rationalizable for type $t_{i}$ if and only if

$$
t_{i} \in C_{i}^{x_{1}, x_{2}}(T)
$$

It is useful to note that, since $R_{i}^{1}=B_{i}^{1-x_{i}}(T), C^{1-x_{1}, 1-x_{2}}(T)$ corresponds to a high common belief in the event that action invest is rational. Hence, each part of the proposition states that an action $a_{i}$ is rationalizable for a type $t_{i}$ if and only if $t_{i}$ assigns sufficiently high probability on a sufficiently high common belief in the event that $a_{i}$ is rational. That is, he finds it sufficiently likely that the action is rational, finds it sufficiently likely that the other player finds it sufficiently likely that the action is rational,..., ad infinitum. The key innovation that yields such a simple characterization is allowing the threshold for the sufficiency to depend on the payoffs of the types throughout.

\section{Risk-Dominant Selection and Multiplicity}

Our focus in this paper is on when both actions are rationalizable for both players and when one action is uniquely rationalizable for both players - without loss of generality, we focus on uniqueness of action invest. Building on the characterization in the previous section, we present intuitive sufficient conditions for each case. Our first result characterizes the cases with multiplicity and uniqueness, as an immediate corollary to the characterization in the previous section.

Corollary 1 Both actions are rationalizable for a type $t_{i}$ if and only if

$$
t_{i} \in C_{i}^{x_{1}, x_{2}}(T) \cap C_{i}^{1-x_{1}, 1-x_{2}}(T) .
$$


Invest is the uniquely rationalizable action for a type $t_{i}$ if and only if

$$
t_{i} \in C_{i}^{1-x_{1}, 1-x_{2}}(T) \backslash C_{i}^{x_{1}, x_{2}}(T) .
$$

The first part characterizes the cases with multiple rationalizable solutions. It states that both actions are rationalizable if and only if there is sufficiently high common belief that both actions are rational for both players. The second part characterizes the cases in which invest is the only rationalizable solution. It states that invest is uniquely rationalizable if and only if there is sufficiently high common belief in rationality of invest (i.e., $t_{i} \in C_{i}^{1-x_{1}, 1-x_{2}}(T)$ ) but there is not sufficiently high common belief in rationality of not invest (i.e., $t_{i} \notin C_{i}^{x_{1}, x_{2}}(T)$ ). Once again, we obtain such simple and straightforward characterizations by making the threshold for sufficiency type-dependent.

While such characterizations are useful conceptually, they may not be of great practical use. In the rest of this Section, we provide simple tractable sufficient conditions for multiplicity and uniqueness. We start with a result for multiplicity, which states that both actions are rationalizable whenever there is approximate common certainty that payoffs support multiple strict equilibria.

Proposition 2 For any $\varepsilon \in[0,1 / 2]$, both actions are rationalizable on $C^{1-\varepsilon, 1-\varepsilon}\left(M_{\varepsilon}\right)$ where

$$
M_{\varepsilon}=\left\{\left(t_{1}, t_{2}\right) \mid \varepsilon \leq x_{i}\left(t_{i}\right) \leq 1-\varepsilon \text { for both } i\right\}
$$

indeed, there exist Bayesian Nash equilibria ${ }^{7} s^{*}$ and $s^{* *}$ such that

$$
s^{*}(t)=(\text { invest, } \text { invest }) \text { and } s^{* *}(t)=(\text { not invest, not invest }) \quad\left(\forall t \in C^{1-\varepsilon, 1-\varepsilon}\left(M_{\varepsilon}\right)\right) .
$$

Proof. We will construct an equilibrium $s^{*}$ as in the Proposition; construction of $s^{* *}$ is identical. We construct an auxiliary game by altering the payoffs of some types as follows. We set $x_{i}\left(t_{i}\right)=2$ whenever $x_{i}\left(t_{i}\right)>1$ and set $x_{i}\left(t_{i}\right)=-1$ whenever $x_{i}\left(t_{i}\right)<0$, so that dominant actions remain dominant. For types in $C^{1-\varepsilon, 1-\varepsilon}\left(M_{\varepsilon}\right)$, we assign payoff 1 for invest and 0 for not invest, making invest strictly dominant on $C^{1-\varepsilon, 1-\varepsilon}\left(M_{\varepsilon}\right)$. The auxiliary game - with bounded supermodular payoffs - satisfies the sufficient conditions of van Zandt (2010) and therefore has a Bayesian Nash equilibrium $s^{*}$, in which the types in $C^{1-\varepsilon, 1-\varepsilon}\left(M_{\varepsilon}\right)$ must play invest. But $s^{*}$ is also a Bayesian Nash equilibrium in the original game, as we show next. Indeed, for each type $t_{i} \in C_{i}^{1-\varepsilon, 1-\varepsilon}\left(M_{\varepsilon}\right)$, equilibrium action $s_{i}^{*}\left(t_{i}\right)=$ invest is a best response to $s_{-i}^{*}$ because $x_{i}\left(t_{i}\right) \geq \varepsilon\left(t_{i} \in M_{\varepsilon}\right.$ by Lemma 1$)$ and type $t_{i}$ assigns at least probability $1-\varepsilon$ on $C_{j}^{1-\varepsilon, 1-\varepsilon}\left(M_{\varepsilon}\right)$ (by Lemma 1) where $s_{j}^{*}$ takes the value of invest throughout. All the remaining types play a best response because their best responses are identical in the original and the auxiliary games.

\footnotetext{
${ }^{7}$ By a Bayesian Nash equilibrium, we mean a strategy profile $s: T \rightarrow\{\text { invest, not invest }\}^{2}$ such that $s_{i}\left(t_{i}\right)$ is a best response to $s_{-i}$ for type $t_{i}$ for each $t_{i}$.
} 
Note that, if the payoffs were known and as in $M_{\varepsilon}$, then both (invest, invest) and (not invest, not invest) would have been $(1-\varepsilon)$-dominant Nash equilibria. Proposition 2 then states that both actions are played in an equilibrium (and hence rationalizable) whenever there is common $(1-\varepsilon, 1-\varepsilon)$-belief that both actions would have been $(1-\varepsilon)$-dominant Nash equilibrium under complete information. This follows a key observation in the robustness literature, going back to Monderer and Samet (1989) which states that any $p$-dominant equilibrium of a game can be extended to a larger type space in which the original game is $p$-evident.

In an earlier working-paper version, we have used the standard techniques in the robustness literature to prove multiplicity of rationalizable actions. ${ }^{8}$ Such techniques can also be used to prove existence of multiple equilibria under additional technical conditions on the type space. Here, we prove existence of multiple equilibria without additional assumptions by using an equilibrium existence result for supermodular games. Note that, in our proof, we specify the equilibrium actions only on $C^{1-\varepsilon, 1-\varepsilon}\left(M_{\varepsilon}\right)$ and the dominance regions. The equilibrium strategies can be highly complex outside of these regions in the general setup considered here. (In contrast, simple cutoff strategies suffice in the type spaces considered in global games literature.)

We next turn to establishing sufficient conditions under which there is a unique rationalizable action. Our key concept will be approximate uniformity of "rank beliefs", which we now define. We will write $\bar{r}_{i}\left(t_{i}\right)$ for the probability that a player assigns to his payoff parameter being greater than or equal to that of the other player, and $\underline{r}_{i}\left(t_{i}\right)$ for the probability that it is strictly greater. We will refer to these expressions as "rank beliefs" as they reflect the player's belief about his rank if the players are ordered by the payoff parameter. Formally, we define

$$
\begin{aligned}
& \bar{r}_{i}\left(t_{i}\right)=\pi_{i}\left(\left\{t_{j} \mid x_{j}\left(t_{j}\right) \leq x_{i}\left(t_{i}\right)\right\} \mid t_{i}\right), \\
& \underline{r}_{i}\left(t_{i}\right)=\pi_{i}\left(\left\{t_{j} \mid x_{j}\left(t_{j}\right)<x_{i}\left(t_{i}\right)\right\} \mid t_{i}\right),
\end{aligned}
$$

and

$$
r_{i}\left(t_{i}\right)=\left(\bar{r}_{i}\left(t_{i}\right)+\underline{r}_{i}\left(t_{i}\right)\right) / 2 .
$$

We refer to $\bar{r}_{i}\left(t_{i}\right), \underline{r}_{i}\left(t_{i}\right)$, and $r_{i}\left(t_{i}\right)$ as the upper rank belief, the lower rank belief and the rank belief of type $t_{i}$, respectively. When the distribution of $x_{j}$ is atomless according to type $t_{i}$, all these beliefs coincide: $\bar{r}_{i}\left(t_{i}\right)=\underline{r}_{i}\left(t_{i}\right)=r_{i}\left(t_{i}\right)$. We define upper and lower rank beliefs separately in order to deal with point masses. Such point masses may arise, for example, under complete information.

Rank belief of a type $t_{i}$ is uniform if he finds it equally likely that either player's value is higher. Formally, we say that rank belief of a type $t_{i}$ is $\varepsilon$-uniform if

$$
\frac{1}{2}-\varepsilon \leq \underline{r}_{i}\left(t_{i}\right) \leq \bar{r}_{i}\left(t_{i}\right) \leq \frac{1}{2}+\varepsilon .
$$

\footnotetext{
${ }^{8}$ The technique involves setting the actions on $C^{1-\varepsilon, 1-\varepsilon}\left(M_{\varepsilon}\right)$ as desired and allowing the remaining types play a best response.
} 
We write $U R B_{\varepsilon}$ for the set of type profiles $\left(t_{1}, t_{2}\right)$ where both players have $\varepsilon$-uniform rank beliefs:

$$
U R B_{\varepsilon}=\left\{\left(t_{1}, t_{2}\right) \mid \frac{1}{2}-\varepsilon \leq \underline{r}_{i}\left(t_{i}\right) \leq \bar{r}_{i}\left(t_{i}\right) \leq \frac{1}{2}+\varepsilon \text { for each } i\right\} .
$$

We sometimes informally say that rank beliefs are approximately uniform to mean that they are $\varepsilon$-uniform for some sufficiently small $\varepsilon$.

Our second concept is a strict version of risk-dominance. We say that action invest is $\varepsilon$-strictly riskdominant for $t_{i}$ if

$$
x_{i}\left(t_{i}\right)>\frac{1}{2}+\varepsilon
$$

We write $S R D_{\varepsilon}$ for the set of type profiles for which invest is $\varepsilon$-strictly risk-dominant for each player, so

$$
S R D_{\varepsilon}=\left\{\left(t_{1}, t_{2}\right) \mid x_{i}\left(t_{i}\right)>\frac{1}{2}+\varepsilon \text { for each } i\right\} .
$$

We say that action invest is strictly risk-dominant if invest is $\varepsilon$-strictly risk-dominant for $\varepsilon=0$.

Proposition 3 For any $\varepsilon \geq 0$, assume that $C^{1-\varepsilon, 1-\varepsilon}\left(U R B_{\varepsilon}\right)$ is closed. Then, invest is the uniquely rationalizable action for both players if it is $2 \varepsilon$-strictly risk-dominant for both players and there is common $(1-\varepsilon)$-belief of $\varepsilon$-uniform rank beliefs, i.e., if

$$
\left(t_{1}, t_{2}\right) \in S R D_{2 \varepsilon} \cap C^{1-\varepsilon, 1-\varepsilon}\left(U R B_{\varepsilon}\right) .
$$

Proposition 3 provides a useful sufficient condition for uniqueness, identifying common features of the uniqueness results in the global games literature. It states that invest is uniquely rationalizable if it is strictly risk-dominant and there is approximate common certainty of approximately uniform rank beliefs. Observe that our result establishes this result without explicitly assuming some of the critical features of global games, such as existence of dominance regions. Moreover, it allows arbitrary type spaces with minimal continuity and compactness properties. In the next section, we will present the proof of our result and further discuss the assumption that the set $C^{1-\varepsilon, 1-\varepsilon}\left(U R B_{\varepsilon}\right)$ is closed. In the rest of this section, we will present some applications of our result. We start with presenting a weaker sufficient condition:

Corollary 2 For any $\varepsilon>0$, let

$$
D U R B_{\varepsilon}=\left\{\left(t_{1}, t_{2}\right) \mid x_{i}\left(t_{i}\right) \in[0,1] \Rightarrow \frac{1}{2}-\varepsilon \leq \underline{r}_{i}\left(t_{i}\right) \leq \bar{r}_{i}\left(t_{i}\right) \leq \frac{1}{2}+\varepsilon \quad \forall i\right\}
$$

be the event that each player has either $\varepsilon$-uniform beliefs or a dominant action. Assume that $C^{1-\varepsilon, 1-\varepsilon}\left(D U R B_{\varepsilon}\right)$ is closed. Then, invest is uniquely rationalizable for both players on $S R D_{2 \varepsilon} \cap C^{1-\varepsilon, 1-\varepsilon}\left(D U R B_{\varepsilon}\right)$. 
That is, invest is uniquely rationalizable whenever it is strictly risk-dominant for both and there is approximate common certainty that each player has either approximately uniform rank beliefs or a dominant action. Note that this sufficient condition merges the uniform rank beliefs and strict risk-dominance properties into a single condition. We simply drop the restriction on rank beliefs when a player has a dominant strategy. This will clearly not matter for strategic results. The corollary immediately follows from applying Proposition 3 to an augmented game in which the beliefs of the types with a dominant action is modified so that they have uniform rank beliefs (by adding new types as necessary).

Our results have immediate applications in low dimensional type spaces, which are often used in applications. Here, we will illustrate a couple of them. In the first two examples, the type spaces will be unidimensional, i.e.,

$$
T_{1}=T_{2}=\mathbb{R}
$$

and in all of them the payoff functions will be linear, i.e.,

$$
x_{i}=t_{i}+y,
$$

where $y \in \mathbb{R}$ is the ex-ante mean of the payoffs.

Unidimensional, Linear-Normal Model In the previous framework, we specify

$$
\begin{aligned}
t_{i} & =\theta+\sigma e_{i} \\
\theta & =\tau \eta
\end{aligned}
$$

where $\sigma$ and $\tau$ positive real numbers and $\eta, e_{1}$, and $e_{2}$ are independent standard normal random variables. Here, each player's payoff has a common component $\theta$ and an idiosyncratic component $e_{i}$. Using the standard formulas, one can compute that the rank belief of a player with type $t$ is

$$
r_{\sigma, \tau}(t)=\Phi\left(\sqrt{\frac{\sigma^{2}}{\left(\sigma^{2}+2 \tau^{2}\right)\left(\sigma^{2}+\tau^{2}\right)}} t\right),
$$

where $\Phi$ is the cumulative distribution function of standard normals; recall that player assigns probability $r_{\sigma, \tau}(t)$ to the event that the other player's type is lower than his own. ${ }^{9}$ What can we say about higher-order beliefs in this case? Observe that, as $\sigma \rightarrow 0$ and $\tau \rightarrow 0, \sqrt{\frac{\sigma^{2}}{\left(\sigma^{2}+2 \tau^{2}\right)\left(\sigma^{2}+\tau^{2}\right)}}$ converges to $\infty$ if $\frac{\sigma}{\tau^{2}} \rightarrow \infty$ and converges to 0 if $\frac{\sigma}{\tau^{2}} \rightarrow 0$. Thus if $\frac{\sigma}{\tau^{2}} \rightarrow \infty$, we obtain approximate common certainty of payoffs and we have limit multiplicity. If $\frac{\sigma}{\tau^{2}} \rightarrow 0$, we do have pointwise convergence to uniform rank beliefs, so that $r_{\sigma, \tau}(t) \rightarrow \frac{1}{2}$ for each $t$. But this convergence is not uniform in the tails, so that for any $(\sigma, \tau), r_{\sigma, \tau}(t) \rightarrow 1$ as $t \rightarrow \infty$ and $r_{\sigma, \tau}(t) \rightarrow 0$ as $t \rightarrow-\infty$. Thus there is never

\footnotetext{
${ }^{9}$ This known formula for rank-beliefs has been extensively used in the existing literature, e.g., Morris and Shin (2001) and Morris and Shin (2003a).
} 
approximate common certainty of approximately uniform rank beliefs. ${ }^{10}$ However, as established in Corollary 2 , it is enough that all types without a dominant actions have approximately uniform rank beliefs. Since $r_{\sigma, \tau}(t)$ approaches to $1 / 2$ uniformly over $[-\bar{y}, 1-\bar{y}]$, when $\frac{\sigma}{\tau^{2}}$ is small, this condition is satisfied, and risk dominance arises as the unique rationalizable outcome.

Unidimensional Linear Model with Fat-tailed Distributions In the previous model assume instead that the random variables $\eta, e_{1}$, and $e_{2}$ have regularly-varying tails where the idiosyncratic terms $e_{1}$ and $e_{2}$ have tail index $\alpha$ and the common term $\eta$ has tail index $\beta$. That is, the tail of $e_{i}$ is approximately proportional to $e_{i}^{-\alpha}$ and the tail of $\eta$ is approximately proportional to $\eta^{-\beta}$. Such models arise often when the players face model uncertainty. When $\alpha>\beta$, the idiosyncratic term has a thinner tail, and the player attributes all large deviations from the mean to a large common shock, assigning nearly probability $1 / 2$ to the event that the other player's type is below his own. In that case, $r_{\sigma, \tau}(t)$ is approximately $1 / 2$ near the tail. Indeed, in an earlier version of this paper, we have shown that we have common knowledge of approximate uniform rank beliefs whenever $\alpha>\beta+1$ and $\sigma / \tau$ is sufficiently small. In that case, Proposition 3 establishes that whenever an action is strictly risk-dominant, it is the unique rationalizable action. Conversely, if $\beta>\alpha$, then the common term has thinner tails, and the players attribute all large deviations from the mean to idiosyncratic shocks in their signals, believing that the other player's type is near the ex-ante mean. Consequently, we have approximate common certainty of the payoffs, and Proposition 2 establishes multiple equilibrium outcomes whenever the payoffs support multiple strict equilibria under complete information. Thus we find that the fat-tailed model delivers sharper sufficient conditions for uniqueness than the well known normal model does.

Linear-Normal Model with Precision Uncertainty In the unidimensional linear normal model, assume that $e_{i} \sim N\left(0, v_{i}^{2}\right)$ where the variance $v_{i} \in[\underline{v}, \bar{v}]$ is privately known by player $i$, and $v_{1}$ and $v_{2}$ are strictly positive and independently distributed with cumulative distribution functions $F_{1}$ and $F_{2}$, respectively, for some $\bar{v}>\underline{v}>0$. Hence, $T_{1}=T_{2}=\mathbb{R} \times[\underline{v}, \bar{v}]$. Then,

$$
r_{\sigma, \tau}\left(t_{i}, v_{i}\right)=E\left[\Phi\left(\sqrt{\frac{\sigma^{2} v_{i}^{2}}{\left(\sigma^{2} v_{j}^{2}+\left(1+v_{j}^{2} / v_{i}^{2}\right) \tau^{2}\right)\left(\sigma^{2} v_{i}^{2}+\tau^{2}\right)}} t_{i}\right) \mid t_{i}, v_{i}\right] .
$$

Observe that, as $\sigma / \tau^{2}$ approaches 0 , the expression in the square root goes to 0 for each $\left(v_{1}, v_{2}\right)$, as in the case with known variances. Since $\Phi$ is bounded, this implies that $r_{\sigma, \tau}\left(t_{i}, v_{i}\right)$ also goes to $\Phi(0)=1 / 2$, yielding approximately uniform rank beliefs for each $\left(t_{i}, v_{i}\right)$. Once again, the convergence

\footnotetext{
${ }^{10}$ For any $\varepsilon<1 / 4$ and $(\sigma, \tau)$, since $\lim _{t \rightarrow \infty} r_{\sigma, \tau}(t)=1$, if the closed set $C^{1-\varepsilon, 1-\varepsilon}\left(U R B_{\varepsilon}\right)$ were not empty, then it would have a maximum $\bar{t}$. But since $\bar{t} \in U R B_{\varepsilon}$, type $\bar{t}$ would assign at most probability $r_{\sigma, \tau}(\bar{t}) \leq 1 / 2+\varepsilon<1-\varepsilon$, contradicting that $\bar{t} \in C^{1-\varepsilon, 1-\varepsilon}\left(U R B_{\varepsilon}\right)$.
} 
is not uniform, but this is not an issue since the variances $v_{1}$ and $v_{2}$ are bounded: for sufficiently small $\sigma / \tau^{2}$, the condition in Corollary 2 is satisfied, and we have risk-dominance as the unique rationalizable outcome.

Interestingly, when $v_{i}$ is not bounded, the rank beliefs can vary arbitrarily regardless of the size of $\sigma / \tau^{2}$. Indeed,

$$
\lim _{v_{i} \rightarrow \infty} r_{\sigma, \tau}\left(t_{i}, v_{i}\right)=E\left[\Phi\left(\sqrt{\frac{1}{\left(\sigma^{2} v_{j}^{2}+\tau^{2}\right)} t_{i}}\right) \mid t_{i}\right] .
$$

Hence, when $v_{i}$ is large and $\sigma$ and $\tau$ are small, $r_{\sigma, \tau}\left(t_{i}, v_{i}\right)$ is approximately 1 for $t_{i}>0$ and 0 for $t_{i}<0$. Nevertheless, one can still obtain common belief of $D U R B_{\varepsilon}$ at any given type when $\sigma / \tau^{2}$ is sufficiently small. To see this, take any $\varepsilon>0$ and any $\left(\left(t_{1}, v_{1}\right),\left(t_{2}, v_{2}\right)\right)$. Set $\bar{v}_{i}(\varepsilon)=\max \left\{F_{i}^{-1}(1-\varepsilon), v_{i}\right\}$ for each $i$, and note that the simple event $E_{\varepsilon}=\mathbb{R} \times\left(0, \bar{v}_{1}(\varepsilon)\right] \times \mathbb{R} \times\left(0, \bar{v}_{2}(\varepsilon)\right]$ is $(1-\varepsilon, 1-\varepsilon)$-evident. Moreover, as in the case of bounded variances, when $\sigma / \tau^{2}$ is sufficiently small, we have $E_{\varepsilon} \subseteq D U R B_{\varepsilon}$. Thus, $E_{\varepsilon} \subseteq C^{(1-\varepsilon, 1-\varepsilon)}\left(D U R B_{\varepsilon}\right)$.

The exercise in the unidimensional linear-normal model is a standard exercise in the global games literature (Morris and Shin (2001) and Morris and Shin (2003a)). In this case, one can explicitly compute rank beliefs and see how they evolve as the distributions shrink to zero at different rates. However, the other two models highlight the fact that these results are special. The unidimensional model with fattailed distributions identifies distinct properties that give uniqueness and multiplicity depending on the tail properties of the distributions. In the model with precision uncertainty, one can easily compute the rank beliefs, but that does not help in analyzing the equilibria per se, because the type space is two dimensional and the event that players invest is a complex event depending on both payoff $t_{i}$ and variance $v_{i}$ (and we cannot compute it). Nevertheless, we can still apply our result and extend the conclusions from the standard exercise to this case.

\section{Proof and a Counterexample}

In this Section, we will present the proof of Proposition 3. A key technical assumption in this Proposition is that common belief of uniform rank beliefs is a closed set. This closure assumption holds for free in three cases:

1. there is common knowledge that beliefs are approximately uniform, i.e., $U R B_{\varepsilon}=T$;

2. the upper and lower rank beliefs are continuous (by Lemma 1); 
3. $\pi_{i}\left(E_{j} \mid t_{i}\right)$ is continuous in $t_{i}$ for each $E_{j}$, and $\pi_{i}\left(\left\{t_{i}\right\} \mid t_{i}\right)=0$ for each $t_{i}{ }^{11}$

We first establish by example that this assumption is not superfluous, illustrating difficulties one would face even under the common certainty of uniform rank beliefs.

Example 1 Consider the following symmetric type space. For some $\hat{t} \in(0,1 / 2)$ and $y=1 / 2$, set

$$
\begin{aligned}
T_{i} & =\mathbb{R} \\
\pi_{i}\left(\cdot \mid t_{i}\right) & = \begin{cases}U\left[t_{i}+\left(\left|t_{i}\right|-\hat{t}\right) / 2, t_{i}+\left(\hat{t}-\left|t_{i}\right|\right) / 2\right] & \text { if } t_{i} \in(-\hat{t}, \hat{t}) \\
\delta_{t_{i}} & \text { otherwise }\end{cases} \\
x_{i}\left(t_{i}\right) & =t_{i}+y,
\end{aligned}
$$

where $U X$ is the uniform distribution on $X$ and $\delta_{t_{i}}$ assigns probability 1 on $t_{j}=t_{i}{ }^{12}$ The range of $t_{j}$ is plotted as a function of $t_{i}$ in Figure 1. This type space satisfies our general assumptions: the beliefs and payoffs are continuous functions of types, and $x_{i}^{-1}([a, b])$ is compact for every interval $[a, b]$. Nonetheless, the rank beliefs are discontinuous:

$$
\bar{r}_{i}\left(t_{i}\right)=1-\underline{r}_{i}\left(t_{i}\right)= \begin{cases}1 / 2 & \text { if } t_{i} \in(-\hat{t}, \hat{t}) \\ 1 & \text { otherwise. }\end{cases}
$$

Clearly, the rank beliefs are uniform on $(-\hat{t}, \hat{t})$ :

$$
U R B_{\varepsilon}=(-\hat{t}, \hat{t})^{2} \quad(\forall \varepsilon \in[0,1 / 2)) .
$$

Moreover, $U R B_{\varepsilon}$ is an evident event: for any $\left(t_{1}, t_{2}\right) \in(-\hat{t}, \hat{t})^{2}$, each player assigns probability 1 on $(-\hat{t}, \hat{t})^{2}$, as vividly demonstrated in Figure 1. Therefore,

$$
C^{1,1}\left(U R B_{\varepsilon}\right)=U R B_{\varepsilon}=(-\hat{t}, \hat{t})^{2} .
$$

That is, we have common certainty of uniform rank beliefs throughout $(-\hat{t}, \hat{t})^{2}$. Note however that $0<$ $x_{i}\left(t_{i}\right)<1$ on $(-\hat{t}, \hat{t})$. Hence, for $\varepsilon=0$, Proposition 2 implies that we have multiplicity over $C^{1,1}\left(U R B_{\varepsilon}\right)$, contradicting the conclusion of Proposition 3.

Example 1 shows that even assuming common certainty of uniform rank beliefs would not be enough without the closure assumption. Example 1 also shows that we cannot dispense with our general technical

\footnotetext{
${ }^{11}$ In that case, the upper and lower rank beliefs coincide, and they are continuous. This assumption holds when $\pi_{i}\left(\cdot \mid t_{i}\right)$ has a bounded density $f\left(\cdot \mid t_{i}\right)$ that is continuous in $t_{i}$.

${ }^{12}$ We could obtain a similar counterxample with a common prior by putting uniform distribution on the convex hull of $\{(-\hat{t},-\hat{t}),(\hat{t}, \hat{t}),(-\bar{t}, \bar{t}),(\bar{t},-\bar{t})\}$ for some small but positive $\bar{t}$ and also on the diagonal outside of that set. We would consider $U R B_{\varepsilon}$ for $\varepsilon=\bar{t} /(2 \hat{t})$.
} 


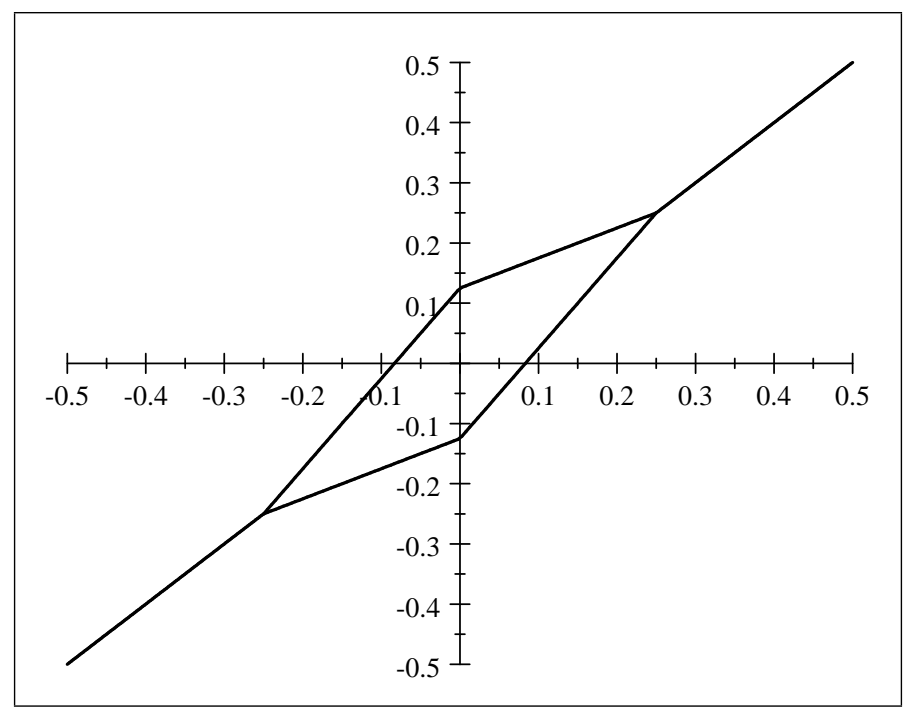

Figure 1: The range of $t_{j}$ as a function of $t_{i}$ for $\hat{t}=1 / 4$.

assumption that the preimage $x_{i}^{-1}([a, b])$ of any compact interval $[a, b]$ is compact. Indeed, one can take $T=(-\hat{t}, \hat{t})^{2}$, giving common certainty that beliefs are uniform, without altering the set of multiple solutions.

Note that on the boundary of the event $U R B_{\varepsilon}=(-\hat{t}, \hat{t})^{2}$, we must have $\bar{r}_{i}(\hat{t})=1$ and $\underline{r}_{i}(\hat{t})=0$, so that $\hat{t} \notin U R B_{\varepsilon}$, rendering $C^{1,1}\left(U R B_{\varepsilon}\right)=U R B_{\varepsilon}$ not closed. As we will show momentarily, this is what leads to multiplicity within $C^{1,1}\left(U R B_{\varepsilon}\right)$. Indeed, as we show below, when $C^{1-\varepsilon, 1-\varepsilon}\left(U R B_{\varepsilon}\right)$ is closed, the set of payoffs are unbounded on $C^{1-\varepsilon, 1-\varepsilon}\left(U R B_{\varepsilon}\right)$, violating the hypothesis of Proposition 2, and allowing contagion from the dominance regions included within $C^{1-\varepsilon, 1-\varepsilon}\left(U R B_{\varepsilon}\right)$.

Note that the sufficient condition for uniqueness in Proposition 3 is local, in that it only refers to the hierarchy of the beliefs of the type at hand, without making global assumptions about the type space. In contrast, the existing works on global games usually make structural assumptions on the entire type space, and often rely on the extremal equilibria. As illustrated in Example 1, a subset of types, such as $(-\hat{t}, \hat{t})^{2}$, can form a belief-closed subspace where the hierarchies do not have any meaningful connection to the rest of the type space. Such hierarchies may not have substantial amount of information about the types outside the subspace. Therefore, our local conditions do not lead to global structural conditions in general. It turns out that when $C^{1-\varepsilon, 1-\varepsilon}\left(U R B_{\varepsilon}\right)$ is a topologically closed set, we can use rank beliefs to deduce enough structure within the set $C^{1-\varepsilon, 1-\varepsilon}\left(U R B_{\varepsilon}\right)$ to obtain the desired result, as we do next.

Fix a closed $p$-evident event $E$ and define two cutoffs:

$$
\begin{aligned}
x^{*}(E) & =\inf \left\{z \mid z=x_{i}\left(t_{i}\right) \geq \underline{r}_{i}\left(t_{i}\right) \text { for some } i \text { and } t_{i} \in E_{i}\right\} \\
x^{* *}(E) & =\sup \left\{z \mid z=x_{i}\left(t_{i}\right) \leq \bar{r}_{i}\left(t_{i}\right) \text { for some } i \text { and } t_{i} \in E_{i}\right\} .
\end{aligned}
$$


Here, $x^{*}(E)$ is the lowest value for which any type of either player within $E$ has that value and has a lower lower-rank belief; and $x^{* *}(E)$ is the highest value for which any type of any player within $E$ has that value and a higher upper-rank belief. The next Lemma establishes that invest cannot be rationalizable when the value is lower than $x^{*}(E)$, and not invest cannot be rationalizable when the value exceeds $x^{* *}(E)$.

Lemma 3 Let $E$ be a closed p-evident event. Then, invest is uniquely rationalizable for any $t_{i} \in E_{i}$ with $x_{i}\left(t_{i}\right)>x^{* *}(E)+(1-p)$ and not invest is uniquely rationalizable for any $t_{i} \in E_{i}$ with $x_{i}\left(t_{i}\right)<$ $x^{*}(E)-(1-p)$.

Proof. We will show that invest is not rationalizable for any $t_{i} \in E_{i}$ with $x_{i}\left(t_{i}\right)<x^{*}(E)-(1-p)$. Let

$$
\hat{x}_{i} \equiv \inf \left\{x_{i}\left(t_{i}\right) \mid t_{i} \in R_{i}^{\infty} \cap E_{i}\right\}
$$

and assume without loss that

$$
\widehat{x}_{1} \leq \widehat{x}_{2} .
$$

When $R_{1}^{\infty} \cap E_{1}$ is empty, our conclusion is vacuously true because invest is not rationalizable anywhere on $E$. We will assume that $R_{1}^{\infty} \cap E_{1}$ is not empty and show that $x^{*}(E)-(1-p) \leq \hat{x}_{1}$. Now, by definition, there exists a sequence $\left(t_{1, m}\right)$ of types $t_{1, m} \in R_{1}^{\infty} \cap E_{1}$ such that $x_{1}\left(t_{1, m}\right) \in\left[\hat{x}_{1}, \hat{x}_{1}+1\right]$ for each $m$ and $x_{1}\left(t_{1, m}\right) \rightarrow \hat{x}_{1}$. Since $x_{1}^{-1}\left(\left[\hat{x}_{1}, \hat{x}_{1}+1\right]\right)$ is sequentially compact, there then exists a convergent subsequence with some limit $\hat{t}_{1}$. Since $E$ is closed, $\hat{t}_{1} \in E_{1}$. Since $x_{1}$ is continuous and $x_{1}\left(t_{1, m}\right) \rightarrow \hat{x}_{1}$, we have

$$
\hat{x}_{1}=x_{1}\left(\hat{t}_{1}\right) .
$$

Now, since $T$ is closed and $x_{i}$ is continuous, by Lemma 2 and Proposition $1, R^{\infty}$ is closed, and hence $\hat{t}_{1}$ remains in the closed set $R_{1}^{\infty} \cap E_{1}$. In particular, $\hat{t}_{1} \in E_{1}$, and

$$
x_{1}\left(\hat{t}_{1}\right) \geq 1-\pi_{1}\left(R_{2}^{\infty} \mid \hat{t}_{1}\right) .
$$

Moreover, since $E$ is $p$-evident, $\pi_{1}\left(R_{2}^{\infty} \backslash E_{2} \mid \hat{t}_{1}\right) \leq \pi_{1}\left(T_{2} \backslash E_{2} \mid \hat{t}_{1}\right) \leq 1-p$. Hence,

$$
\pi_{1}\left(R_{2}^{\infty} \mid \hat{t}_{1}\right) \leq \pi_{1}\left(R_{2}^{\infty} \cap E_{2} \mid \hat{t}_{1}\right)+(1-p) .
$$

Therefore,

$$
\begin{aligned}
\hat{x}_{1} & =x_{1}\left(\hat{t}_{1}\right), \text { by }(4) \\
& \geq 1-\pi_{1}\left(R_{2}^{\infty} \mid \hat{t}_{1}\right), \text { by }(5) \\
& \geq 1-\pi_{1}\left(R_{2}^{\infty} \cap E_{2} \mid \hat{t}_{1}\right)-(1-p), \text { by }(6) \\
& \geq 1-\pi_{1}\left(\left\{t_{2} \in T_{2} \mid x_{2}\left(t_{2}\right) \geq \hat{x}_{2}\right\} \mid \hat{t}_{1}\right)-(1-p), \text { by definition of } \widehat{x}_{2} \\
& \geq 1-\pi_{1}\left(\left\{t_{2} \in T_{2} \mid x_{2}\left(t_{2}\right) \geq \hat{x}_{1}\right\} \mid \hat{t}_{1}\right)-(1-p), \text { by }(3) \\
& =\pi_{1}\left(\left\{t_{2} \in T_{2} \mid x_{2}\left(t_{2}\right)<x_{1}\left(\hat{t}_{1}\right)\right\} \mid \hat{t}_{1}\right)-(1-p) \\
& =\underline{r}_{1}\left(\hat{t}_{1}\right)-(1-p),
\end{aligned}
$$


showing that

$$
\widehat{x}_{2} \geq \widehat{x}_{1} \geq x^{*}(E)-(1-p) .
$$

A symmetric argument establishes that not invest is not rationalizable if $x_{i}\left(t_{i}\right)>x^{* *}(E)+(1-p)$.

When $x^{* *}(E)=1$ (or $x^{*}(E)=0$ ), Lemma 3 is vacuous, stating that invest is uniquely rationalizable when it is dominant. When $x^{* *}(E)<p$, Lemma 3 establishes that invest remains uniquely rationalizable throughout the interval $\left(x^{* *}(E)+1-p, 1\right)$.

This is similar to the main result of Carlsson and van Damme (1993). In their result, if one can connect a type $t_{i}$ to a type $\bar{t}_{i}$ at which invest is a dominant action, via a continuous path along which invest is either risk-dominant or dominant, then invest is uniquely rationalizable at $t_{i}$. Here, as in Example 1 , such an assumption may not be useful, and we do not explicitly make any such assumption. Nonetheless, when $x^{* *}(E)<p$, each type $t_{i} \in E_{i}$ with $x^{* *}(E)<x_{i}\left(t_{i}\right)<1$ assigns a substantial probability (i.e. a probability greater than $1-x_{i}\left(t_{i}\right)$ ) on a set of types $t_{j}$ whose values are higher than that of $t_{i}$ and assign a substantial probability to yet another set of types with similar properties. In general, such connections through belief hierarchies do not necessarily lead to a contagion path. Indeed, as illustrated in Example 1, such a path can remain within the open set $(-\hat{t}, \hat{t})^{2}$, without leading to a dominance region. However, when $E$ is closed and $p>\max _{t_{i} \in E_{i}} \bar{r}\left(t_{i}\right)$, such a chain necessarily leads to types who assign a substantial probability on types for which invest is a dominant action. ${ }^{13}$ Such a chain forms a contagion path. Note that the types in the chain have type-dependent thresholds as in Proposition 1.

Proof of Proposition 3. Proposition 3 immediately follows from Lemma 3. Since $C^{1-\varepsilon, 1-\varepsilon}\left(U R B_{\varepsilon}\right) \subseteq$ $U R B_{\varepsilon}$, we clearly have $x^{* *}\left(C^{1-\varepsilon, 1-\varepsilon}\left(U R B_{\varepsilon}\right)\right) \leq 1 / 2+\varepsilon$. Hence, whenever $\left(t_{1}, t_{2}\right) \in S R D_{2 \varepsilon} \cap C^{1-\varepsilon, 1-\varepsilon}\left(U R B_{\varepsilon}\right)$, we have $x_{i}\left(t_{i}\right)>1 / 2+2 \varepsilon \geq x^{* *}\left(C^{1-\varepsilon, 1-\varepsilon}\left(U R B_{\varepsilon}\right)\right)+\varepsilon$. Since $C^{1-\varepsilon, 1-\varepsilon}\left(U R B_{\varepsilon}\right)$ is $(1-\varepsilon)$-evident, Lemma 3 then implies that invest is uniquely rationalizable for both players at $\left(t_{1}, t_{2}\right)$.

\section{Discussion: Extensions and Relation to The Literature}

\subsection{Non-Uniform Rank Beliefs}

We have focussed on the case of uniform rank beliefs, which is the leading case that arises in the literature. Our results continue to hold if we have common certainty that players have rank belief $p$, i.e., they assign probability $p$ to having the higher rank. Our uniqueness results extend immediately, although the nature of the selection is then different.

Specifically, define $R B_{\varepsilon}^{p}$ for the set of type profiles $\left(t_{1}, t_{2}\right)$ where both players have $\varepsilon$-rank belief $p$ :

$$
R B_{\varepsilon}^{p}=\left\{\left(t_{1}, t_{2}\right) \mid p-\varepsilon \leq \underline{r}_{i}\left(t_{i}\right) \leq \bar{r}_{i}\left(t_{i}\right) \leq p+\varepsilon \text { for each } i\right\} .
$$

\footnotetext{
${ }^{13}$ Indeed, one can show that when $C^{p, p}\left(U R B_{\varepsilon}\right)$ is closed for some $p>1 / 2+\varepsilon$, the set $x\left(C^{p, p}\left(U R B_{\varepsilon}\right)\right)$ of payoff parameters for the types within $C^{p, p}\left(U R B_{\varepsilon}\right)$ is unbounded, inducing dominance regions within the subspace.
} 
Say that action invest is strictly $p$-dominant for $t_{i}$ if

$$
x_{i}\left(t_{i}\right)>1-p
$$

We write $D^{p}$ for the set of type profiles for which invest is strictly $p$-dominant for each player, so

$$
D^{p}=\left\{\left(t_{1}, t_{2}\right) \mid x_{i}\left(t_{i}\right)>1-p \text { for each } i\right\} .
$$

Proposition 4 For any $p, q, \varepsilon \in[0,1]$ with $p+q \geq 1-2 \varepsilon$, assume that $C^{1-\varepsilon, 1-\varepsilon}\left(R B_{\varepsilon}^{q}\right)$ is closed. Then, invest is the uniquely rationalizable action for both players if it is strictly p-dominant for both players and there is common $(1-\varepsilon)$-belief of $\varepsilon$-rank belief $q$, i.e., if

$$
\left(t_{1}, t_{2}\right) \in D^{p} \cap C^{1-\varepsilon, 1-\varepsilon}\left(R B_{\varepsilon}^{q}\right) .
$$

This observation, suitably generalized to many players, is the driving force behind the results of Izmalkov and Yildiz (2010). The following example illustrates how approximate common certainty of non-uniform rank beliefs can arise.

Unidimensional Linear Model with Exponential Distributions Assume $\sigma=\tau=1$,

$$
\begin{aligned}
x_{i} & =t_{i}+y \\
t_{i} & =\theta+e_{i}
\end{aligned}
$$

where $y<0$ and

$$
\theta, e_{1}, e_{2} \stackrel{i i d}{\sim} \operatorname{Exp}(\lambda)
$$

Observe that, conditional on $t_{i}, \theta$ is uniformly distributed on $\left[0, t_{i}\right]$; so is $e_{i}$. Hence,

$$
r\left(t_{i}\right)=\frac{1}{t_{i}} \int_{0}^{t_{i}}\left(1-e^{-\lambda\left(t_{i}-\theta\right)}\right) d \theta=1-\frac{e^{-\lambda t_{i}}}{t_{i}} \int_{0}^{t_{i}} e^{\lambda \theta} d \theta=1-\frac{1-e^{-\lambda t_{i}}}{\lambda t_{i}}
$$

The rank beliefs are plotted in Figure 2. As $\lambda \rightarrow \infty$,

$$
r\left(t_{i}\right) \rightarrow 1
$$

uniformly on $[-y, \infty)$. Thus, if $\bar{t}_{\varepsilon}=r^{-1}(1-\varepsilon)$ and $E_{\varepsilon}=\left\{t_{1} \mid t_{1} \geq \bar{t}_{\varepsilon}\right\} \times\left\{t_{2} \mid t_{2} \geq \bar{t}_{\varepsilon}\right\}$, the event $E_{\varepsilon}$ is $(1-\varepsilon, 1-\varepsilon)$-evident.

The example satisfies the common prior assumption. One could build on the example to show that invest could be made the unique rationalizable action. It thus establishes that approximate common certainty of non-uniform rank beliefs is consistent with the unique selection of any rationalizable action in a type that is close to complete information in the product topology, illustrating a result of Weinstein and Yildiz (2007). However, approximate common certainty of non-uniform rank beliefs cannot arise with high ex ante probability under the common prior assumption, illustrating a result of Kajii and Morris (1997). 


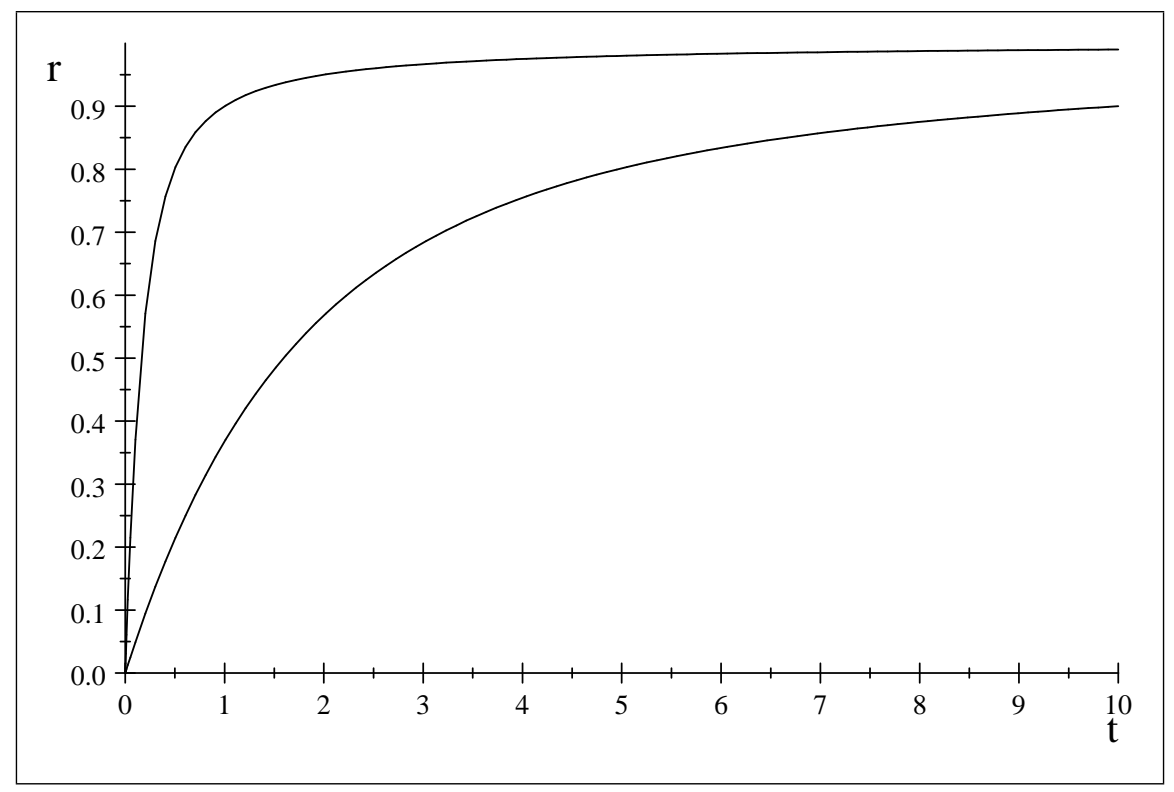

Figure 2: Rank beliefs for $\lambda=1$ and $\lambda=10$.

\subsection{Many Players}

We focussed on the case of two players. The extension of the results of this paper to $N$ players - maintaining the symmetry and separability of payoffs assumptions in this paper - is straightforward, and we describe this extension in this subsection.

Suppose that each player $i$ has a payoff type $x_{i}$, the payoff to not investing is 0 , and the payoff to investing is $x_{i}-1+\psi(l)$ where $l$ is the proportion of other players investing and $\psi:[0,1] \rightarrow[0,1]$ is increasing with $\psi(0)=0$ and $\psi(1)=1$. In the special case of two players, these payoffs reduce to those in the body of this paper.

We can give a common belief characterization of rationalizability in this setting, although the relevant belief operator is more complicated than in the two player case. For a fixed simple event $E$, agent $i$ and integer $n$, we can consider derived events corresponding to the set of type profiles $t_{-i}$ of players other than $i$ where $t_{j} \in E_{j}$ for exactly $n$ out of those other players. We will be interested in the probability that player $i$ assigns to such derived events and, more specifically, whether a weighted sum of such probabilities is above some type-dependent level. Thus we define generalized belief operators:

$$
B_{i}^{f_{i}}(E)=\left\{t \mid t_{i} \in E_{i} \text { and } \sum_{n=0}^{N-1} \psi\left(\frac{n}{N-1}\right) \pi_{i}\left(\left\{t_{-i} \mid \#\left\{j \mid t_{j} \in E_{j}\right\}=n\right\} \mid t_{i}\right) \geq f_{i}\left(t_{i}\right)\right\} .
$$

This is a generalization of the type-dependent belief operators that we introduced in the two player case. It is qualitatively more complicated, though, because it depends on the weighted sum of probabilities 
assigned to a set of events (rather than probabilities of one event). However, with these operators, we can generalize the tight characterization of rationalizability. For a vector of type dependent probability functions $f=\left(f_{1}, . ., f_{N}\right)$, we can now define $f$-belief and common $f$-belief operators as before,

$$
\begin{gathered}
B_{*}^{f}(E)=\cap_{i=1}^{N} B_{i}^{f_{i}}(E) . \\
C^{f}(E)=\cap_{n=1}^{\infty}\left[B_{*}^{f}\right]^{n}(E) .
\end{gathered}
$$

and analogous fixed point characterizations will hold. Now Lemma 2 will continue to hold as stated for these modified operators, as will Proposition 1 giving a sufficient condition for a uniquely rationalizable action in symmetric games. It is also possible to give generalized belief operator characterizations of rationalizable actions in more general games, see Morris and Shin (2007).

While the tight characterization of rationalizability becomes more complicated when we move to many players, the sufficient conditions we give for multiplicity and uniqueness generalize straightforwardly. The approximate common certainty sufficient condition for multiplicity in Proposition 2 is sufficient to show rationalizability of all strict equilibrium actions in general games, as originally shown by Monderer and Samet (1989).

For uniqueness sufficient conditions, a player's rank belief now gives the probability that he assigns to his payoff type being ranked $k$ th for each $k$, and we can define corresponding upper and lower rank beliefs. Thus

$$
\begin{aligned}
& \bar{r}_{i}\left(k \mid t_{i}\right)=\pi_{i}\left(\#\left\{t_{j} \mid x_{j}\left(t_{j}\right) \leq x_{i}\left(t_{i}\right)\right\}=k \mid t_{i}\right), \\
& \underline{r}_{i}\left(k \mid t_{i}\right)=\pi_{i}\left(\#\left\{t_{j} \mid x_{j}\left(t_{j}\right)<x_{i}\left(t_{i}\right)\right\}=k \mid t_{i}\right) .
\end{aligned}
$$

We say that rank belief of a type $t_{i}$ is $\varepsilon$-uniform if

$$
\frac{1}{N}-\varepsilon \leq \underline{r}_{i}\left(k \mid t_{i}\right) \leq \bar{r}_{i}\left(k \mid t_{i}\right) \leq \frac{1}{N}+\varepsilon
$$

for each $k=1, . ., N$. We write $U R B_{\varepsilon}$ for the set of type profiles $t$ where both players have $\varepsilon$-uniform rank beliefs:

$$
U R B_{\varepsilon}=\left\{t \mid \frac{1}{N}-\varepsilon \leq \underline{r}_{i}\left(n \mid t_{i}\right) \leq \bar{r}_{i}\left(n \mid t_{i}\right) \leq \frac{1}{N}+\varepsilon \text { for each } i\right\}
$$

We say that rank beliefs are approximately uniform if they are $\varepsilon$-uniform for some sufficiently small $\varepsilon \geq 0$.

Morris and Shin (2003b) noted that the global game selection in this case was the "Laplacian action", corresponding to a uniform belief over the proportion of opponents investing. Our second concept is a strict version of the Laplacian property. We say that action invest is $\varepsilon$-Laplacian for $t_{i}$ if

$$
x_{i}\left(t_{i}\right) \geq 1-\frac{1}{N} \sum_{j=0}^{N-1} \psi\left(\frac{j}{N-1}\right)+\varepsilon .
$$


We write $L_{\varepsilon}$ for the set of type profiles for which invest is $\varepsilon$-strictly Laplacian for each player, so

$$
L_{\varepsilon}=\left\{t \mid x_{i}\left(t_{i}\right) \geq 1-\frac{1}{N} \sum_{j=0}^{N-1} \psi\left(\frac{j}{N-1}\right)+\varepsilon \text { for each } i\right\} .
$$

Proposition 5 For any $\varepsilon \geq 0$, assume that $C^{1-\varepsilon, 1-\varepsilon}\left(U R B_{\varepsilon}\right)$ is closed. Then, invest is the uniquely rationalizable action for all players if it is $\varepsilon$-Laplacian for all players and there is common $(1-\varepsilon)$-belief of $\varepsilon$-uniform rank beliefs, i.e., if

$$
t \in L_{2 \varepsilon} \cap C^{1-\varepsilon}\left(U R B_{\varepsilon}\right)
$$

The generalized belief operators described above have been used by Oyama and Takahashi (2013) to prove results about robustness to incomplete information games in the sense of Kajii and Morris (1997). There is a continuum of players in much of the global games literature. Extending our analysis, as stated, to a continuum of players would raise new technical issues, e.g., concerning the metrizability of the type space. Two papers that do use explicit statements about higher-order beliefs to give sufficient conditions for uniquely rationalizable actions in games with a continuum of players are Morris and Shin (2012) and Morris (2014). However, they are established in much more restrictive settings than this paper.

\subsection{Global Games Literature}

Let us briefly summarize how the results described in this paper relate to the existing global games literature.

The classical exercise in the global games literature initiated by Carlsson and van Damme (1993) is to consider what happens if we fix a prior over payoff relevant states and let the size of the noise in players' conditionally independent signals of the state converge to zero. A key step in such arguments (e.g., in Carlsson and van Damme (1993) and Frankel, Morris, and Pauzner (2003)) is the assumption that the prior distribution is smooth, which ensures that conditional probabilities converge uniformly, or uniformly over a compact interval. In the symmetric case, globally uniform convergence of conditional probabilities gives approximate common certainty of approximately uniform rank beliefs. However, as highlighted by Lemma 2, it is enough to have common certainty of rank beliefs on a compact interval including states where players actions are undominated. In this sense, we highlight the properties of higher-order beliefs that drive results in the symmetric case.

Our results generalize those in the existing literature because we do not exploit monotonicity properties of the type space. An order structure on types is used in the existing literature. In the analysis of Carlsson and van Damme (1993), Morris and Shin (2003b) and Frankel, Morris, and Pauzner (2003), there is monotonicity with respect to types in the limit (as noise goes to zero) and continuity arguments are used to provide results in the (not necessarily) monotonic type space away from the limit. In the normal models 
of Morris and Shin (2001) and Morris and Shin (2003a), monotonicity away from the limit is implied by the normality. In highlighting the connection between general supermodular games and global games, van Zandt and Vives (2007) impose monotonicity away from the limit in general games. Mathevet (2010) imposes a stochastic dominance property even in the limit to prove global game uniqueness results via a contraction argument. By contrast, results in this paper are proved without any order structure on types.

\section{A Omitted Proofs}

Proof of Lemma 2. (Part 1) If $\left(t_{1}, t_{2}\right) \in B_{*}^{\left(f_{1}, f_{2}\right)}(E)$, then, for each $i$, we have $t_{i} \in B_{i}^{\left(f_{1}, f_{2}\right)}(E)$, implying $t_{i} \in E_{i}$. Therefore, $B_{*}^{\left(f_{1}, f_{2}\right)}(E) \subseteq E$. By iterative application of this, one gets the statement in Part 1 .

(Part 2) For any $\left(f_{1}, f_{2}\right)$-evident $F \subseteq E$, we have

$$
F \subseteq C^{\left(f_{1}, f_{2}\right)}(F) \subseteq C^{\left(f_{1}, f_{2}\right)}(E),
$$

where the first equality is by definition of $\left(f_{1}, f_{2}\right)$-evident, and the second inequality is by monotonicity of probability distributions. ${ }^{14}$ From Part 1, it then suffices to show that $C^{\left(f_{1}, f_{2}\right)}(E)$ is $\left(f_{1}, f_{2}\right)$-evident. To this end, take any $\left(t_{1}, t_{2}\right) \in C^{\left(f_{1}, f_{2}\right)}(E)$. For each $n$ and $i$, since $\left(t_{1}, t_{2}\right) \in C^{\left(f_{1}, f_{2}\right)}(E) \subseteq\left[B_{*}^{\left(f_{1}, f_{2}\right)}\right]^{n+1}(E)$, we have

$$
\pi_{i}\left(\left[B_{*}^{\left(f_{1}, f_{2}\right)}\right]_{j}^{n}(E) \mid t_{i}\right) \geq f_{i}\left(t_{i}\right)
$$

where $\left[B_{*}^{\left(f_{1}, f_{2}\right)}\right]_{j}^{n}(E)$ is the projection of $\left[B_{*}^{\left(f_{1}, f_{2}\right)}\right]^{n}(E)$ to $T_{j}$. But, by definition and part $1,\left[B_{*}^{\left(f_{1}, f_{2}\right)}\right]_{j}^{n}(E)$ is a decreasing sequence (indexed by $n$ ) converging to $C_{j}^{\left(f_{1}, f_{2}\right)}(E)$, the projection of $C^{\left(f_{1}, f_{2}\right)}(E)$ to $T_{j}$. Therefore,

$$
\pi_{i}\left(C_{j}^{\left(f_{1}, f_{2}\right)}(E) \mid t_{i}\right)=\lim _{n} \pi_{i}\left(\left[B_{*}^{\left(f_{1}, f_{2}\right)}\right]_{j}^{n} \mid t_{i}\right) \geq f_{i}\left(t_{i}\right),
$$

showing that $\left(t_{1}, t_{2}\right) \in B_{i}^{f_{i}}\left(C^{\left(f_{1}, f_{2}\right)}(E)\right)$ and proving that $C^{\left(f_{1}, f_{2}\right)}(E)$ is $\left(f_{1}, f_{2}\right)$-evident.

(Part 3) It suffices to show that $B_{i}^{f_{i}}(E)$ is closed; the rest follows from the fact that arbitrary intersections of closed sets are closed. Take any sequence $t_{i, m} \rightarrow t_{i}$, where $\left(t_{i, m}, t_{j}\right) \in B_{i}^{f_{i}}(E)$ for some $t_{j}$, so that $\pi_{i}\left(E_{j} \mid t_{i, m}\right) \geq f_{i}\left(t_{i, m}\right)$ for each $m$. Then, since $E_{i}$ is closed, $t_{i} \in E_{i}$, and, by the Portmanteau Theorem, we have

$$
\pi_{i}\left(E_{j} \mid t_{i}\right) \geq \lim \sup \pi_{i}\left(E_{j} \mid t_{i, m}\right) \geq \lim f_{i}\left(t_{i, m}\right)=f_{i}\left(t_{i}\right)
$$

showing that $\left(t_{i}, t_{j}\right) \in B_{i}^{f_{i}}(E)$.

\section{References}

Argenziano, R. (2008): "Differentiated networks: Equilibrium and Efficiency," The RAND Journal Of Economics, 39, 747-769.

\footnotetext{
${ }^{14}$ Indeed, if $\left(t_{1}, t_{2}\right) \in B_{i}^{f_{i}}(F)$, then $t_{i} \in F_{i} \subseteq E_{i}$ and $\pi_{i}\left(E_{j} \mid t_{i}\right) \geq \pi_{i}\left(F_{j} \mid t_{i}\right) \geq f_{i}\left(t_{i}\right)$, showing that $\left(t_{1}, t_{2}\right) \in B_{i}^{f_{i}}(E)$.
} 
Aumann, R. (1976): "Agreeing to Disagree," Annals of Statistics, 4, 1236-1239.

Carlsson, H., and E. van Damme (1993): "Global Games and Equilibrium Selection," Econometrica, 61, 9891018.

Frankel, D., S. Morris, and A. Pauzner (2003): "Equilibrium Selection in Global Games with Strategic Complementarities," Journal of Economic Theory, 108, 1-44.

Heifetz, A., And Z. Neeman (2006): "On the Generic (Im)Possibility of Full Surplus Extraction in Mechanism Design," Econometrica, 74, 213-233.

Izmalkov, S., And M. Yildiz (2010): "Investor Sentiments," American Economic Journal: Microeconomics, 2, $21-38$.

KAJII, A., AND S. Morris (1997): "The Robustness of Equilibria to Incomplete Information," Econometrica, 65, 1283-1309.

Mathevet, L. (2010): "A Contraction Principle for Finite Global Games," Economic Theory, 42, 539-563.

Mertens, J., And S. Zamir (1985): "Formalization of Bayesian Analysis for Games with Incomplete Information," International Journal of Game Theory, 14, 1-29.

Monderer, D., And D. Samet (1989): "Approximating Common Knowledge with Common Belief," Games and Economic Behavior, 1, 170-190.

Morris, S. (2014): "Coordination, Timing and Common Knowledge," Research in Economics, 68, 306-314.

Morris, S., And H. Shin (1998): "Unique Equilibrium in a Model of Self-Fulfilling Currency Attacks," American Economic Review, 88, 587-597.

Morris, S., ANd H. Shin (2001): "Rethinking Multiple Equilibria in Macroeconomics," NBER Macroeconomics Annual, 2000, 139-161.

Morris, S., And H. Shin (2003a): "Coordination Risk and the Price of Debt," European Economic Review, 48, $133-153$.

(2003b): "Global Games: Theory and Applications," in Advances in Economics and Econometrics: Proceedins of the Eight World Congress of the Econometric Society, ed. by M. Dewatripont, L. Hansen, and S. Turnovsky, pp. 56-114. Cambridge University Press, Cambridge.

(2005): "Heterogeneity and Uniqueness in Interaction Games," in The Economy as an Evolving Complex System, III. Santa Fe Institute.

Morris, S., And H. Shin (2012): "Contagious Adverse Selection," American Economic Journal: Macroeconomics, $4,1-21$.

Morris, S., And H. S. Shin (2007): "Common Belief Foundations in Global Games," Discussion paper, http://www.princeton.edu/ smorris/pdfs/cbf.pdf. 
Oyama, D., And S. Takahashi (2013): "Generalized Belief Operator and the Impact of Small Probability Events on Higher Order Beliefs," Discussion paper, University of Tokyo and National University of Singapore.

VAn ZANDT, T. (2010): "Interim Bayesian Nash Equilibrium on Universal Type Spaces for Supermodular Games," Journal of Economic Theory, 145, 249-263.

van Zandt, T., And X. Vives (2007): "Monotone Equilibria in Bayesian Games of Strategic Complementarities," Journal of Economic Theory, 134, 339-360.

Weinstein, J., And M. Yildiz (2007): "A Structure Theoem for Rationalizability with Applications to Robust Predictions of Refinements," Econometrica, 75, 365-400. 